\title{
Mushroom-Like EBG to Improve Patch Antenna Performance for C-Band Satellite Application
}

\author{
M. K. Abdulhameed ${ }^{1}$, M. S. Mohamad Isa ${ }^{2}$, Z. Zakaria ${ }^{3}$, Mowafak K. Mohsin ${ }^{4}$, Mothana L. Attiah ${ }^{5}$ \\ ${ }_{1,2,3,4,5}$ Centre for Telecommunication Research and Innovation (CeTRI), Faculty of Electronic and Computer Engineering, \\ Universiti Teknikal Malaysia Melaka (UTeM), Hang Tuah Jaya, 76100, Durian Tunggal, Melaka, Malaysia \\ ${ }^{1,4}$ Ministry of Higher Education and Scientific Research, Iraq
}

\section{Article Info}

Article history:

Received Jun 9, 2018

Revised Aug 12, 2018

Accepted Aug 20, 2018

\section{Keyword:}

C-Band satellite

High dielectric constant

High gain

Mushroom-like EBG

ThicK substrate

\begin{abstract}
In order to suppress the surface waves excitation that are caused by thick substrate in a patch antenna, a mushroom-like EBG (Electromagnetic Band Gap) structure is used. Such structures enhance its characteristics of gain, directivity, bandwidth and efficiency. Firstly, we determined frequency band gap characteristics of mushroom like EBG unit cell value by using CST software with $3 \mathrm{~mm}\left(0.06 \lambda_{\mathrm{o}}\right)$ for covering $6 \mathrm{GHz}$. The periodic arrangement of such mushroom-like EBG structures was not limited by any interconnecting microstrip lines. Four columns of EBGs shifted inwards to antenna edges by $0.3 \mathrm{~mm}\left(0.06 \lambda_{o}\right)$ or a gap of its design around the patch from the left and right sides. Different configurations were also examined in order to get the better improvement in antenna performance. The final design of this mushroom-like shifted periodic structure shows an effective increase in the directivity by $77 \%$, gain by $108 \%$, bandwidth by $29 \%$ and the efficiency by $20 \%$ for the antenna. This structure has diversified application possibility for wireless and satellite communications.
\end{abstract}

Copyright $(0) 2018$ Institute of Advanced Engineering and Science. All rights reserved.

\section{Corresponding Author:}

M. K. Abdulhameed,

Department of Telecommunication Engineering,

Faculty of Electronic and Computer Engineering,

Universiti Teknikal Malaysia Melaka,

Hang Tuah Jaya, 76100 Durian Tunggal, Melaka, Malaysia.

Email: eng_mka@yahoo.com

\section{INTRODUCTION}

The microstrip patch antennas have been used widely in various applications due to their inherent benefit of being low profile, low cost, light-weight and their convenient integration with RF devices[1], [2]. Excitation of the surface waves is among the major limitations of these microstrip antennas. The mushroomlike EBG surface described in [3], and the description of the uni-planar EBG surface presented in [4], was utilized for surrounding a microstrip patch antenna to reduce the surface wave impact and improve the antenna performance [5]-[11]. In addition to surface wave suppression support, such structures have been utilized to increase the gain of antenna [12], decreasing the back radiations [13] and reduction in the size of antenna [14].

The high dielectric constant substrate with microstrip antennas are exciting topic in antennas design due to their compressed size. However, utilization of such high dielectric constant substrate brings along some major drawbacks to antenna design. These weaknesses include narrowing of bandwidth, decreased efficiency and inappropriate radiation patterns. These drawbacks originated due to the excitation of surface waves. The amount of energy radiated into air can be less than the energy that is confined by such electromagnetic waves. These surface waves, which are scattered from the edges of antenna can cause back lobe radiation increment, deterioration in gain deterioration and ripples in the radiation pattern [15]. 
Recently, many methods have been introduced to overcome these shortcomings that use the manipulation of antenna substrate. By the use of micromachining techniques, the effective dielectric constant of substrate under the patch can be lowered [16]. This approach has a major shortcoming that the larger patch size than that on the unperturbed substrate. The surface wave can be lowered by putting a complete band-gap structure around the patch [5] or by synthesizing low dielectric constant substrate. The high dielectric constant substrate with microstrip antennas resulted in a compressed size but the disadvantage of this method is that the bandwidth of high dielectric constant antennas is narrower than the bandwidth of low dielectric constant. By increasing the thickness of the substrate, this problem can be solved; however this solution will increas the surface waves. In order to overcome the increased surface waves caused by the use of thick substrate, EBG can be used. This approach will give a miniaturized level of both the back and the side lobe, thus there will be an improvement in energy radiation in the direction of broadside. Hence the directivity and gain of antenna will be improved.

In this paper, we applied EBG structure to the patch antenna for the suppression of the surface waves on the high dielectric constant substrate of the antenna. None of the necessary feature, like the bandwidth and small size, was sacrificed, but there is improvement for gain, directivity, bandwidth and efficiency. The EBG structure band gap has been designed to cover the resonant frequency of antenna, thus preventing the surface waves propagation that are originated from antenna. One can improve the performance of antenna by shifting the location of EBG structures and varying their numbers.

\section{EBG STRUCTURE AND DESIGN}

The mushroom-like EBG structure, which is actually a 2-D EBG surface, was initially proposed by [3]. There were four parts in the proposed model: (a) ground plane, (b) dielectric substrate, (c) metallic patches, and (4) connecting vias. A distinct feature of stopband was exhibited by these EBG structures for surface-wave propagation. An LC filter array can be used for the explanation of the operation mechanism of the EBG structure, as shown in Figure 1(c). The current, which is flowing through vias, causes the inductor L effect, whereas the gap among the neighboring patches resulted in the capacitor $\mathrm{C}$ effect. [17] demonstrated a formula to determine the values of $\mathrm{L}$ and $\mathrm{C}$ for an EBG structure, shown in Figure 1(a) and Figure 1(b). W is the patch width, gap width is $\mathrm{g}$, thickness of substrate is $\mathrm{h}$ and $\epsilon_{\mathrm{r}}$ is dielectric constant.

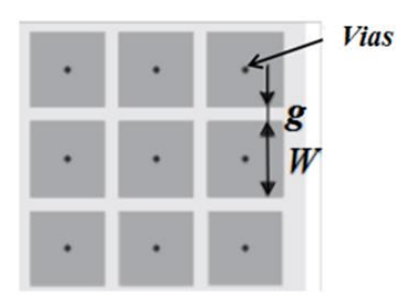

(a) Top view

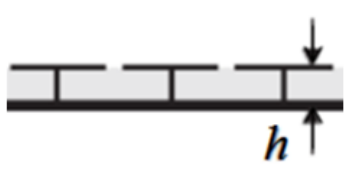

(b) Cross view

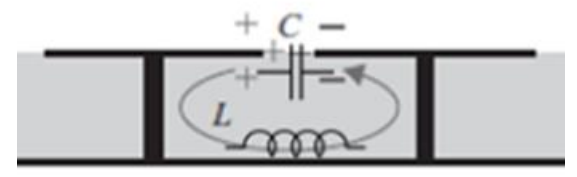

(c) Lumped LC

Figure 1. Geometries of mushroom like EBG (a) Top view (b) Cross view (c) Lumped LC typical for EBG analysis [3]

$$
\begin{aligned}
& L=\mu_{o} h \\
& c=\frac{W \varepsilon_{o}\left(1+\varepsilon_{r}\right)}{\pi} \cosh ^{-1}\left(\frac{2 W+g}{g}\right)
\end{aligned}
$$

Where $\mu_{o}$ is the free space permeability and $\epsilon_{o}$ is the free space permittivity. Likewise we can calculate the frequency band gap as formula below:

$$
W=\frac{1}{\sqrt{L C}}
$$




$$
B W=\frac{\Delta W}{W}=\frac{1}{\eta} \sqrt{\frac{L}{C}}
$$

$\eta$ is the impedance of the free space which is $120 л$ (377 ohms).

The EBG structure operation can be designed by an LC filter equivalent circuit model if periodicity of structure is as small as possible in comparison with the wavelength of operating frequency [18]. The impedance of the structure is very high at resonant frequency, and hence the structure avoids the emission of any surface waves. This results in a band gap of frequency [3]. An increment in patch width $W$, and decrement in gap $g$, while keeping the height and permittivity of substrate fixed, causes an increment in capacitance that results in the decrement of the structure's resonant frequency. To cover the frequency of $6 \mathrm{GHz}, \mathrm{CST}$ software has been used to tune the values of $W$ and $g$. Figure 2(a) demonstrate the unit cell of the mushroom-EBG with $W=3 \mathrm{~mm}\left(0.06 \lambda_{\mathrm{o}}\right), g=0.3 \mathrm{~mm}\left(0.006 \lambda_{\mathrm{o}}\right)$ and via diameter of $1 \mathrm{~mm}\left(0.02 \lambda_{\mathrm{o}}\right)$ connected between the ground plane and patch on a Rogers RT/Duroid 6010 substrate of height $h=2.2 \mathrm{~mm}$ $\left(0.044 \lambda_{\mathrm{o}}\right)$, relative permittivity of 10.2 . The working EBG band width defines as the frequency range within which the reflection phase changes from $-\pi / 2$ to $+\pi / 2$ as shown in Figure 2(b), so the resonant frequency $(6 \mathrm{GHz})$ at the zero reflection phases. The key idea of this research is to suitably design the patch size such that the antenna resonant frequency drops within the EBG band gap and then the surface waves could be prohibited. The band gaps of $3 \mathrm{~mm}\left(0.06 \lambda_{\mathrm{o}}\right)$ patches are suitable for the side lobe and back radiation reductions, where the antenna frequency drops inside the range area of EBG band gap. In other way, we can state that the EBG band gap can cover the resonance frequency of this designed antenna.

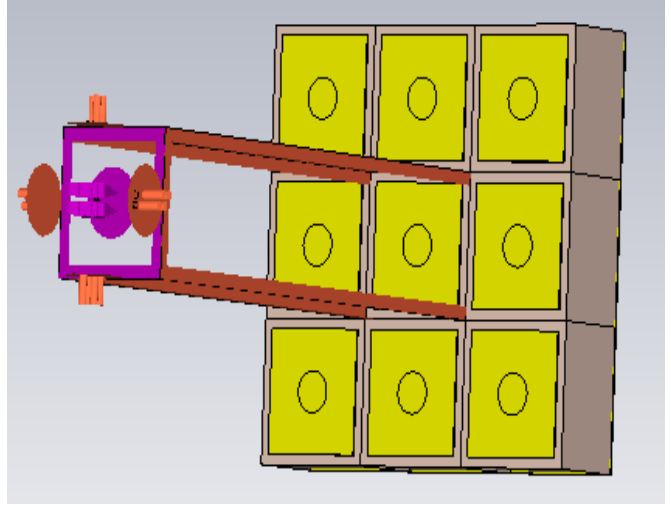

(a)

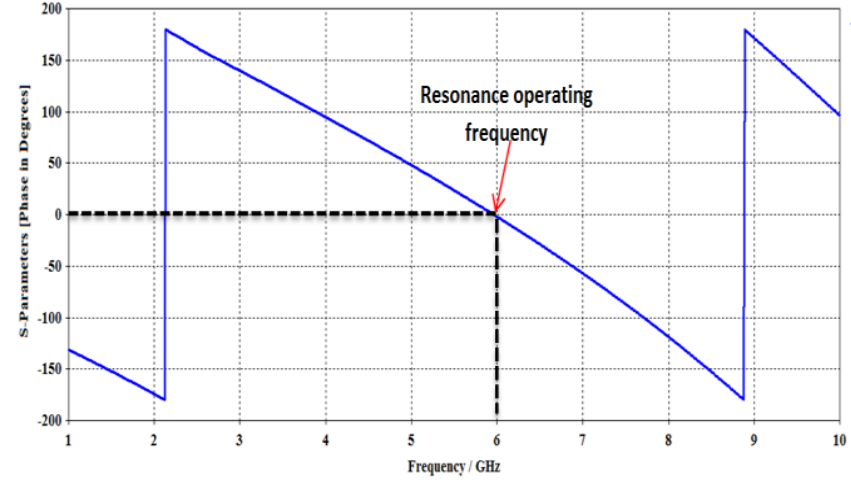

(b)

Figure 2. (a) EBG unit cell (b) Reflection phase for the EBG unit cell

\section{ANTENNA DESIGN AND CONFIGURATION WITH MUSHROOM-LIKE EBG}

Antenna designe has been divided into two main parts, in this work. First, related to design the mushroom-like EBG with frequency band gap that can cover the resonance frequency of the rectangular pach antenna which was desinged and presented in previous part .Configuring the rectangular patch antenna with EBG has given the improvement in antenna performance which is shown in this section.

Patch antenna widely known as microstrip patch antenna and a lot of research has been done by the other investigators to make it usable with a small size. Patch antennas are available in different shapes and sizes. Rectangular patch is a most simple and easy to fabricate. The size of the antenna decreases as the relative dielectric constant of the substrate increase [19]. In this research, we designed a rectangular patch antenna with probe feed on the Rogers RT/Duroid 6010, dielectric constant $\epsilon_{r}=10.2$ and the thickness of the substrate is $h=2.2 \mathrm{~mm}\left(0.044 \lambda_{\mathrm{o}}\right)$. The lengths of the patch are $6.1 \mathrm{~mm}\left(0.124 \lambda_{\mathrm{o}}\right)$ by $7.4 \mathrm{~mm}\left(0.148 \lambda_{\mathrm{o}}\right)$. By surrounding the rectangular patch antenna with 4 columns for all sides, we successfully achieved the antenna improvement by suppressing the surface waves. Structures of EBG were sited more than two periods away from the patch antenna edges. Unlike uni-planar and other EBGs, in mushroom-like structures there are no interconnecting microstrip lines that limit their arrangement periodically. Using this feature, the structures of EBG have been shifted inwards to the antenna ends, so the patch antenna from the left and right sides has been surrounded by 4 columns shifted inwards a gap of its design or $0.3 \mathrm{~mm}\left(0.06 \lambda_{o}\right)$ with overall size is $49.2 \mathrm{~mm}\left(0.98 \lambda_{o}\right) \times 52 \mathrm{~mm}\left(1.04 \lambda_{\mathrm{o}}\right)$ is as be shown in Figure 3 . 


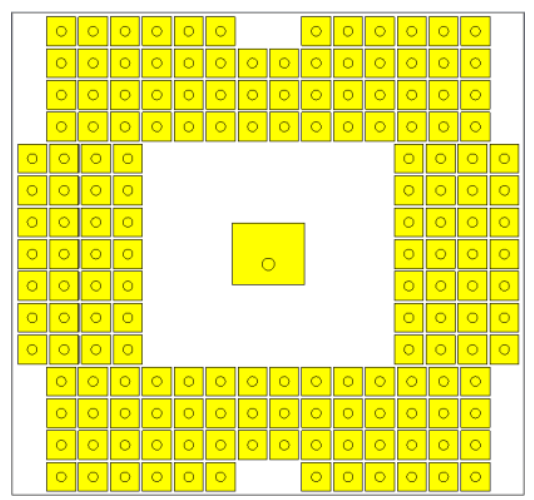

Figure 3. A rectangular patch antenna surrounded by mushroom-like EBG structures

\section{RESULTS AND ANALYSIS}

In the previous section, the design of the rectangular patch antenna, surrounded by the EBG with the band gap which covers $6 \mathrm{GHz}$ frequency was presented. For performance comparison, we simulated antenna surrounded by EBG and and the original rectangular patch antenna. The number of EBG columns that surrounded the antenna is varying from three to five. It is observed that when fewer EBG patch columns (3columns) were used, the improvements in the results were not much significant. As we increased the number of columns to four, with shifting and deleting some of EBG elements as shown in Figure 3, the performance of the structure got enhanced for the patch antenna. This signifies that this structure has a better effect for the antenna resonance frequency. The EBG case with five columns has a relatively larger size and lower improvements were observed than the EGB with four columns. The simulation outcomes are arranged in Table 1 below.

Table 1. Designs Results of EBG on Antenna's Performance

\begin{tabular}{cccccc}
\hline Columns & Directivity $(\mathrm{dBi})$ & Gain $(\mathrm{dB})$ & Efficiency $(\%)$ & S11(dB) & Bandwidth $(\mathrm{MHz})$ \\
\hline (3)columns & 8.33 & 7.82 & $88 \%$ & -20 & 175 \\
(4)columns & 10 & 9.86 & $96.5 \%$ & -31 & 233 \\
(5)columns & 8.4 & 7.9 & $89 \%$ & -31 & 206 \\
Withou tEBG & 5.66 & 4.74 & $80 \%$ & -20.7 & 181 \\
\hline
\end{tabular}

The simulated S-parameter in term of S-11 presented a good matching S-11 at $6 \mathrm{GHz}$. The configurations slightly shifted the resonant frequency of the antenna to $5.98 \mathrm{GHz}$ when using 4-shifted columns for both sides of antenna (right and left) with good matching S-11 of less than $-31 \mathrm{~dB}$. While the resonance frequency for the antenna without using EBG pointed at $6.01 \mathrm{GHz}$ with matching $\mathrm{S}-11$ equal to $-20.7 \mathrm{~dB}$ as shown in Figure 4.

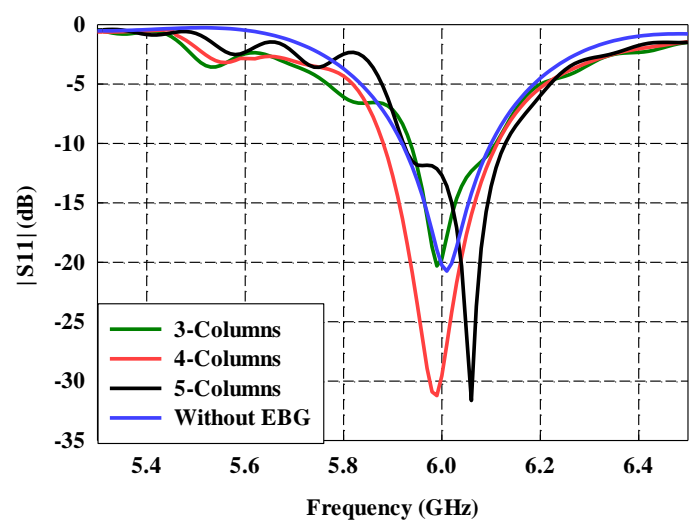

Figure 4. Return loss of the microstrip antennas surrounded by different columns of EBG structures 
In order to compare the performance of antenna, the EBG structured antenna was adjusted to resonant frequency at $6 \mathrm{GHz}$ and this is compared with rectangular patch antenna without using EBG. The radiation patterns for the directivity of both antennas is as demonstrated in Figure 5(a).

The rectangular patch antenna without EBG has $-7 \mathrm{~dB}$ side lobes. This side lobe causes a reduction in the antenna gain. Additionally; the main lobe has a beam width of around 87.3 . This big aperture angle would cause a reduction in the directivity of antenna. The electric field radiation properties of the antenna with four shifted columns of EBG observed the better reduction in side lobe level around $-17.5 \mathrm{~dB}$. Further, the beam width is about $57^{\circ}$. These features provided a better gain and directivity in comparison to the rectangular patch antenna with no EBG. It has been noticed that when the antenna is surrounded by the mushroom-like EBG structures, a reduction in the side lobes and back radiation could be observed and there is an increment in terms of gain in the direction of broadside of the antenna. The measured gain of a rectangular patch antenna with EBG elements is $9.86 \mathrm{~dB}$ and the directivity is $10 \mathrm{dBi}$, while the gain and directivity for the rectangular antenna without using EBG are $4.74 \mathrm{~dB}$ and $5.66 \mathrm{dBi}$ as shown in Figure 5(b) and Figure 5(c).

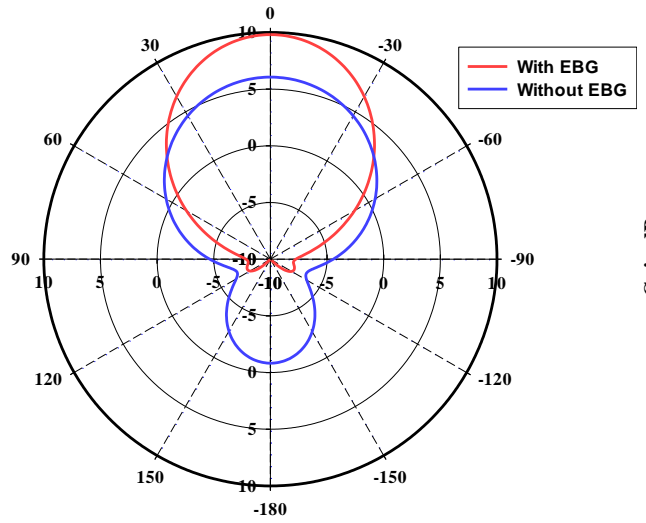

(a)

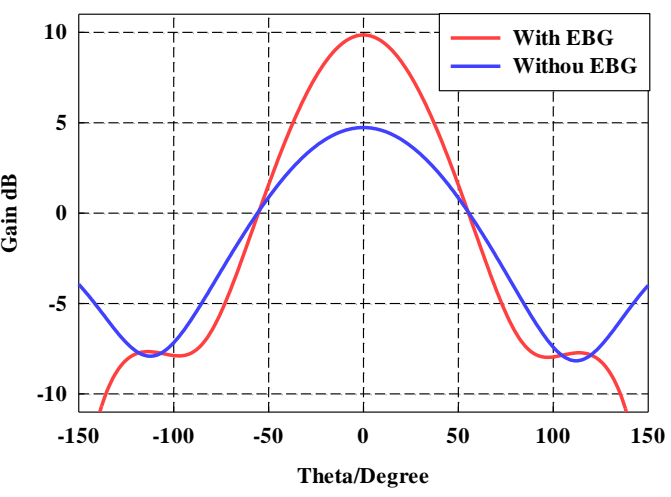

(b)

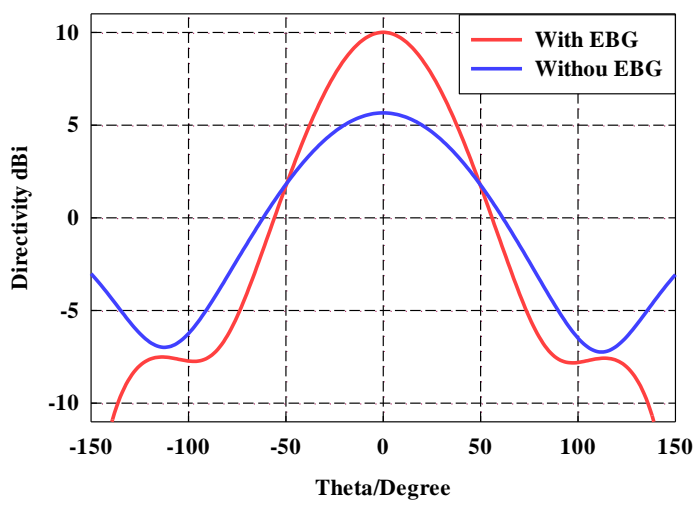

(c)

Figure 5. (a) Radiation pattern improvement with EBG (b) Increase gain (c) Increase directivity

A surface wave effect in radiation pattern is often considered undesirable, since it decrease the antenna efficiency and causes an increment in the side lobe. By increasing the substrate thickness to avoid the narrow band width due to the high dielectric constant, the surface waves will also increase and this problem can be solved by using EBG for the suppression of such surface waves. Figure 6 shows an improvement in the efficiency of antenna when the EBG was used to surround the rectangular antenna. Antenna without EBG has efficiency of $80 \%$ and the efficiency has been observed to be improved to $96.5 \%$ after the EBG in shifted columns configurations was applied on the very same antenna. 


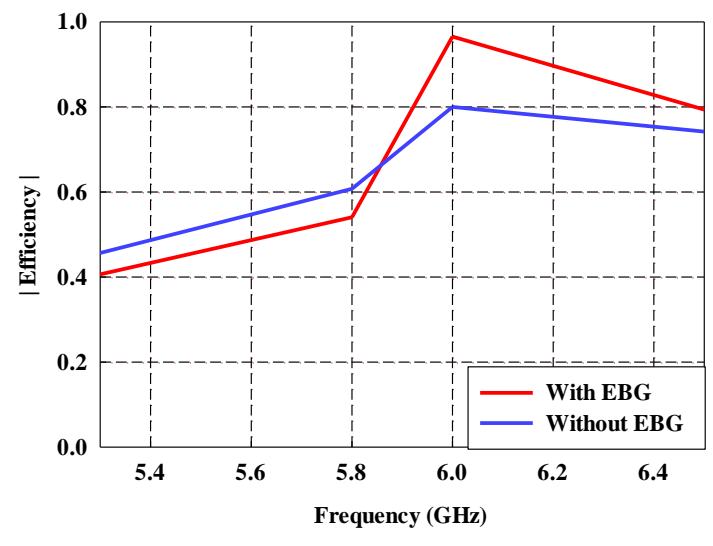

Figure 6. Efficiency improvement by using EBG

\section{CONCLUSION}

This paper presented a novel arrangement of the mushroom-like EBG structure to avoid the bad effects of surface wave produced by the high permittivity and thick substrate without losing the small size of antenna. The new design shows an enhancement in the gain, directivity, bandwidth and efficiency of antenna as compared to the original rectangular patch antenna. The placement of mushroom-like structures is flexible which provide yet another advantage in the applications of current design of antenna. Various results are demonstrating their ability to develop the patch antenna performance on high dielectric constant substrate. The final design represents a good improvement in the antenna performance where the directivity is improved by $77 \%$ (from $5.66 \mathrm{dBi}$ to $10 \mathrm{dBi}$ ), the realized gain also showed a high improvement of $108 \%$ (from $4.74 \mathrm{~dB}$ to $9.86 \mathrm{~dB}$ ), the bandwidth increased by $29 \%$ (from $181 \mathrm{MHz}$ to $233 \mathrm{MHz}$ ), and at the same time, the efficiency has been improved from $80 \%$ to $96.5 \%$. The overall antenna size is $49.2 \mathrm{~mm}\left(0.98 \lambda_{o}\right) \times 52 \mathrm{~mm}\left(1.04 \lambda_{o}\right)$. These structures have vast applications in wireless a and satellite communications.

\section{ACKNOWLEDGEMENTS}

This work was supported by UTeM Zamalah Scheme. The authors would also like to thank Center for Research and Innovation Management (CRIM), Centre of Excellence, UTeM, UTeM's research grant JURNAL/2018/FKEKK/J00001 and Universiti Teknikal Malaysia Melaka (UTeM) for their encouragement and help in sponsoring this study.

\section{REFERENCES}

[1] O. Otman, et al., "A New Design of a Wideband Miniature Antenna Array", Int. J. Electr. Comput. Eng., vol. 7, no. 4, p. $1850,2017$.

[2] R. A. Abdulhasan, et al., "Inverted Diamond-shaped Notched Substrate and Patch for High-frequency Interference on Ultra-wideband Antenna", Int. J. Electr. Comput. Eng., vol. 7, no. 6, pp. 2929-2935, 2017.

[3] D. Sievenpiper, et al., "High-impedance Electromagnetic surfaces with a forbidden Frequency Band", IEEE Trans. Microw. Theory Tech., vol. 47, no. 11, pp. 2059-2074, 1999.

[4] F. R. Yang, et al,M "A Uniplanar Compact Photonic-bandgap (UC-PBG) Structure and its Applications for Microwave Circuits", IEEE Trans. Microw. Theory Tech., vol. 47, no. 8, pp. 1509-1514, 1999.

[5] R. Gonzalo, et al., "Enhanced Patch-antenna Performance by Suppressing surface waves using Photonic-bandgap Substrates", IEEE Trans. Microw. Theory Tech., vol. 47, no. 11, pp. 2131-2138, 1999.

[6] J. S. Colburn, "Patch Antennas on Externally Perforated high Dielectric Constant Substrates", IEEE Trans. Antennas Propag., vol. 47, no. 12, pp. 1785-1794, 1999.

[7] A. M. S. Diaz, et al., "Analysis and Design of Electromagnetic Band Gap Structures with Application in Planar Antennas", 2017 42nd Int. Conf. Infrared, Millimeter, Terahertz Waves, pp. 1-2, 2017.

[8] M. S. M. Isa et al., "Antenna Beam Steering using Sectorized Square EBG", J. Telecommun. Electron. Comput. Eng., vol. 4, no. 1, pp. 39-44, 2012.

[9] M. S. Mohamad Isa, et al., "Antenna Pattern Diversity using EBG", 2010 Loughbrgh. Antennas Propag. Conf. LAPC 2010, November, pp. 325-328, 2010.

[10] S. Chen, et al., "Study on Radiation Performances of Dipole Antenna with Diamond-structure EBG Substrate Fabricated by 3D Printing Technique", Int. J. Appl. Ceram. Technol., January, pp. 1-5, 2018.

[11] A. Altaf, M. A. Alsunaidi, and E. Arvas, "A Novel EBG Structure to Improve Isolation in MIMO Antenna, " IEEE, 
pp. 105-106, 2017.

[12] W. Ebg and V. Reflector, "Gain Improvement of MSAs Array by V sing Curved", Int. Electr. Eng. Congr. IEEE, pp. 4-7, 2014.

[13] S. Yamini and B. Panjavarnam, "Microstrip Patch Antenna Integrated with EBG", Proc. 2017 2nd Int. Conf. Comput. Commun. Technol. ICCCT 2017, pp. 41-45, 2017.

[14] J. Zaid, et al., "Miniaturized Microstrip Patch Antenna using Electromagnetic Band Gap (EBG) structures for Multiport Passive UHF RFID-Tag Applications", IEEE, pp. 2459-2460, 2017.

[15] P. Kovács and T. Urbanec, "Electromagnetic Band Gap Structures: Practical Tips and Advice for Antenna Engineers", Radioengineering, vol. 21, no. 1, pp. 414-421, 2012.

[16] G. P. Gauthier, et al., "Microstrip Antennas on Synthesized Low Dielectric-constant Substrates", IEEE Trans. Antennas Propag., vol. 45, no. 8, pp. 1310-1314, 1997.

[17] D. Sievenpiper, High-Impedance Electromagnetic Surfaces, PhD Thesis, p. 150, 1999.

[18] Q. R. Zheng, et al., "A Novel Compact Spiral Electromagnetic Band-gap (EBG) Structure", IEEE Trans. Antennas Propag., vol. 56, no. 6, pp. 1656-1660, 2008.

[19] O. Of and H. M. M. Patch, "Design and Optimization of h-shaped Multi-band Microstrip Patch Antenna", Int. J. Eng. Sci. Manag. Res., vol. 3, pp. 22-26, 2016. 